In this issue of JAOA, two original papers related to AIDS are found, along with the complete text of the surgeon general's report on AIDS. Dr. Koop's report, which was released on October 22, 1986, begins on page $64 / 103$.

"Radiologic manifestations of acquired immunodeficiency syndrome," by Pera, Weisgrau, Harle, and Rios, is an excellent and comprehensive review. The various radiologic presentations of the disease have been compiled according to the three body systems most affected by AIDS - the central nervous system, the gastrointestinal system, and the pulmonary system. The authors point out that radiologic manifestations are often the initial findings in AIDS. The report begins on page 48/87.

"Psychiatric aspects of AIDS: Overview for the general practitioner," by Johnson, appears on page 60/99. According to Dr. Johnson, it is not surprising that AIDS patients are at special risk for psychiatric illness, given the bleak prognosis, the complex pathophysiology, and the social implications of the disease. In addition to organic brain syndromes, opportunistic infections, and tumors, many patients have pre-existing illnesses that are exacerbated by the diagnosis of AIDS. Dr. Johnson calls for a holistic approach to treatment, and asks that physicians consider the patient's emotional as well as medical needs when applying osteopathic principles to the patient's total treatment plan.

Readers may wish to refer to the December issue of THE Do for additional information on the federal AIDS policy, as well as an in-depth look at how both physicians and patients are confronting the complexities of AIDS. The "Patient Health Guide" in the December issue of the JAOA also tackles the subject in its discussion of "The blood supply in the age of AIDS." This patient-education handout allays fears about blood transfusions by outlining the steps that have been taken to safeguard the blood supply.

THOMAS W. ALLEN, D.O., FACOI

\section{The importance of the negative test}

The January issue of THE Do features a series of information sheets that can be used for patient education. It explains in easy-to-understand terms what some of the more common clinical tests are about, and what to expect when they are performed. The general belief is that the more facts people have, the less they have to fear.

In this era of public and professional emphasis on the cost of medical care, tests that tend to produce negative results are being discouraged. It is dangerous, however, to subscribe to the philosophy that a negative test contributes nothing but cost to the rendering of quality medical care.

Those of us who have been in practice for any length of time soon realize that it is as important to the patient to know what is not wrong as it is to know what is wrong. For example, if a whole battery of tests are performed, a simple statement that "all your tests were negative" does not always allay a person's fears. It is just as easy to say that "your electrocardiogram did not reveal any heart disease, the blood test did not show any evidence of anemia, and the chest $x$-ray did not reveal any abnormality that might have serious consequences." The same approach applies to blood pressure readings. It is amazing how often a patient reports that his physician took his blood pressure but never told him whether it was high or low.

From the patient's standpoint and for the physician who is trying to make a differential diagnosis, the negative laboratory test or $\mathrm{x}$-ray is as important as a positive result. All findings should be conveyed to the patient. As an example, a patient complained of knee pain that had persisted for 2 or 3 months. Although the history and physical examination indicated some degree of chronic medial meniscus pathology, $x$-rays were ordered. They were negative, and the patient was notified. On the spur of the moment, the doctor added that there was no evidence of malignancy. Before the physician could retract what he considered an unnecessary and possibly alarming remark, the patient interjected, "Thank God! The pain had lasted so long-I was sure it was cancer."

The physician's office should be a place where diseases and malfunctions are discovered and treated. But it should also be a place where patients are reassured and given understanding of what is and what is not wrong.

In the practice of quality medicine, there is no basis for disparaging the importance of the negative finding. More importantly, there is no justification for not communicating negative results to a worried patient.

GEORGE W. NORTHUP, D.O., FAAO

\section{$\overline{\text { editorial comment }}$}

The relationship between trace metals and the normal aging process was examined at a recent conference, which was jointly sponsored by the continued on page 21/58 


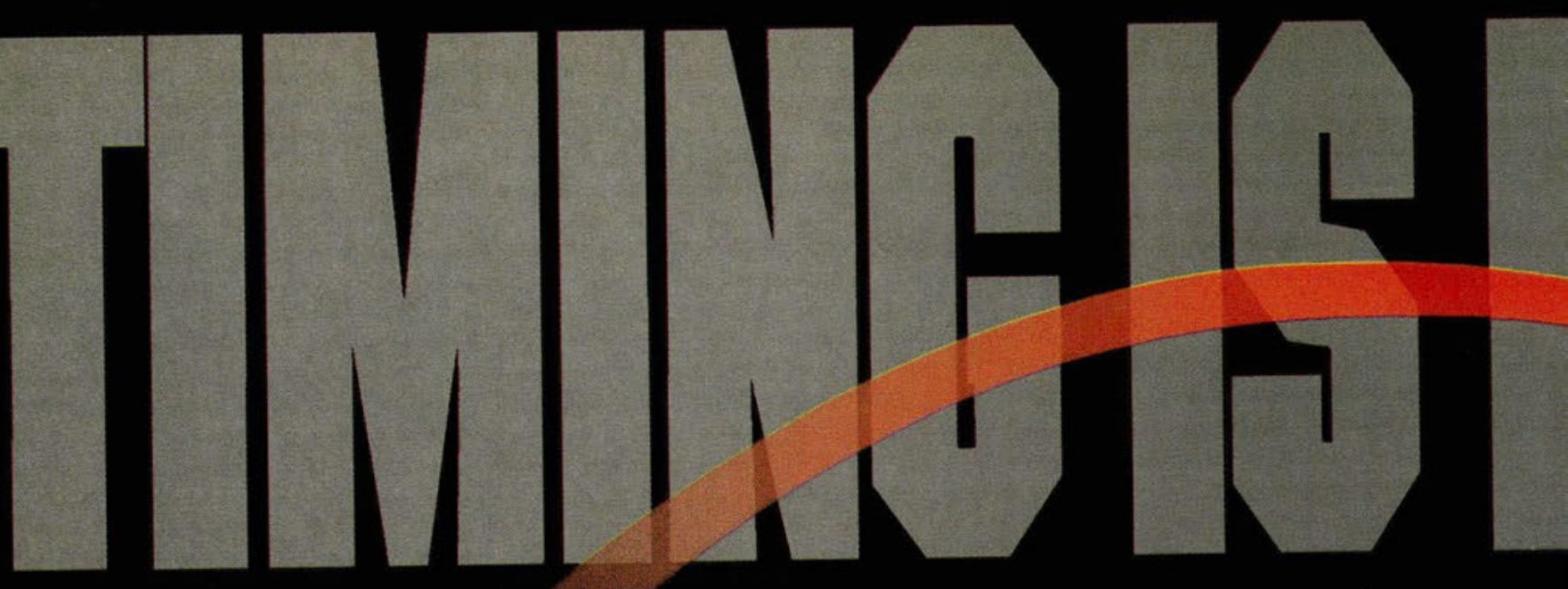


Reference:

1. Sachs R. Frank M. Fishman SK: Overview of clinical experience with glipizide. In Glipizide: A Worldwide Review Princeton, NJ. Excerpta Medica, 1984, pD 163-172.

cucotroto (alipiride) Tablets

\section{Brief Summary of Prescribing Information}

IMDICATIONS with non-insulin-dependent diabetes mellitus (NIDDM, type II) atter an adequate trial of dietary therapy has proved unsatisfactory

. GUCOTROL is contraindicated in patients with known hypersensitivity to the drug of with 政

SPECIAL WARMIMG OM INCREASED RISK OF CARDIOVASCULAR MORTALITY: The administration of orat hypoglycemic drugs has been reported to be associated with increased cardiovascular mortality as compared to treatment with diet alone or diet plus insulin. This warning is based on the study conducted by the University Group Diabetes Program (UGDP), a long-term prospective clinical trial designed to evaluate the effectiveness of glucose-lowering drugs in preventing or delaying vascular complications in patients with non-insulin-depende etes. The study involved

19, supp. 2:747-830, 1970

UCDP reported that patients treated for 5 to 8 years with diet plus a fixed dose of tolbutamide (1.5 grams per day) continued based on the significant increase in totam mor increase in cardiovascular mortily. mortaily. Desplle controversy an adequate basis for this warning. The patient sho
GLUCOTROL and of alternative modes of therapy.

Although only one drug in the sultonylurea class (tolbutamide) was included in this study, it is prudent from a Although only one drug in the sulfonylurea class (tolbutamide) was included in this study, it is prudent from a view of their close similarities in mode of action and chemical structure. PRECAUTIONS: Renal and Hepatic Disease: The metabolism and excretion of GLUCOTROL may be slowed in pat with impaired renal and/or hepatic function. Hypoglycemia may be prolonged in such patients should it occur Hypoglycemia: All sultonylureas are capable of producing severe hypoglycemia. Proper patient selection, dosage. and instructions are important to avoid hypoglycemia. Renal or hepatic insutficiency may increase the risk of hypoglycemic reactions. Elderly, debilitated or malnourished patients and those with adrenal or pituitary insutficiency hypoglycemic reactions. Ele particularty susceptible to the hypoglycemic action of glucose-lowering drugs. Hypoglycemia may be difficult to are particularty susceptible to the hypoglycemic action of glucose the the caloric intake is deficient, after severe or prolonged exercise, when alcohol is ingested, or when more than one caloric intake is deficient, after
glucose-lowering drug is used

glucose-lowering drug is used. A loss of control may occur in diabetic patients exposed to stress such as fever, trauma. infection or surgery. It may then be necessary to discontinue GLUCOTROL and administer insulin

trauma, intection or surgery. ilt may then be necessary to discond

globin may be useful.

列 of therapy, as well as the importance of adhering to dietary instructions, of a regular exercise program, and of regular testing of urine and or blood glucose. The risks of hypoglycemia, its symptoms and realment, and condions that predspose to its development shoine

the

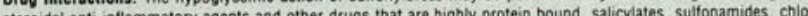
steridal a ramphenicol, pros Howest, caution must be exercised in extrapolating these findings to a clinical situation. Certain drugs tend to However, caution must be exers produce hypersy phenothiazines, thyroid products, estrogens, of ctential interaction between oral miconazole and oral hypoglycemic calculs char topical, or vaginal preparations of miconazole is not known.

Carcin, of Carcinogenesis, muages coses up to 75 timicty tests were uniformily negative. Studies in rats of both sexes at doses up to 75 times the human dose showed no effects on fertility.

. Pro all dose levels $(5.50 \mathrm{mg} / \mathrm{kg})$ This fetotoxicity has been similarly noted with other sultonylureas, such as at all dose ind tolazmide. The eftect is perinatal and believed to be directly related to the pharmacolog torbutamide and tolazamide. TOC (hypoglycemic) action olectudies in pregnant women. GLUCOTROL should be used during pregnancy only if the potential benefit justifies the potential risk to the fetus.

pocaul bent information sugests that abnormal blood glucose levels during pregnancy are associated with because rece ce during pregnancy to higher incidence of congenital as close to normal as possible.

Nonteratogenic EHtects: Prolonged severe hypoglycemia has been reported in neonates born to mothers who were receiving a sultonylurea drug at the time of delivery. This has been reported more frequently with the use of agents with proivo a salt-lives. GLUCOTROL should be discontinued at least one month before the expected delivery date. Nursing Mothers: Since some sultonylurea drugs are known to be excreted in human milik, insulin therapy should be considered it nursing is to be continued

Pediatric Use: Safty and ettectiveness in children have not been established

ADVERSE REACTIONS: In controlled studies, the frequency of serious adverse reactions reported was very low. 0 702 patients. $11.8 \%$ reported adverse reactions and in only $1.5 \%$ was GLUCOTROL discontinued.

Hypoglycemia: See PRECAUTIONS and OVERDOSAGE sections.

Gestrointestinal: Gastrointestinal disturbances, the most common, were reported with the following approximate astrointe: nausea and diarrhea, one in 70 ; constipation and gastralgia, one in 100 . They appear to be dose-related an may disapear on division of reduction of dosage. Chloestatic jaundice may occur rarely with sulfonylureas GLUCOTROL should be discontinued it this occurs.

Dermatologic: Alergic skin reactions including erythema, morbiliform or maculopapular eruptions, urticaria pruritus, and eczema have been reported in about one in 70 patients. These may be transient and may disappear despite continued use of GLUCOTROL, it skin reactions persist, the drug should be discontinued. Porphyria cutane tarda and photosensitivity reactions have been reported with sultonylureas

Hematologic: Leukopenia, agranulocytosis, thrombocytopenia, hemolytic anemia, aplastic anemia, and pan cytopenia have been reported with sulfonylureas.

Metabolic: Hepatic porphyria and disulfiram-like alcohol reactions have been reported with sultonylureas. Clinical experience to date has shown that GLUCOTROL has an extremely low incidence of disulfiram-like reactions.

Endocrine Reactions: Cases of hyponatremia and the syndrome of inappropriate antiduretic hormone (SIADH) secretion have been reported with this and other sultonylureas

Miscellaneous: Dizziness, drowsiness, and headache have been reported in about one in fifty patients treated with GLUCOTROL. They are usually transient and seldom require discontinuance of therapy.

OVERDOSAGE: Overdosage of sulfonylureas including GLUCOTROL can produce hypoglycemia. It hypoglycemic coma is diagnosed or suspected, the patient should be given a rapid intravenous injection of concentrated $(50 \%)$ glucose solution. This should be followed by a continuous infusion of a more dillute $(10 \%)$ glucose solution at rate that will maintain the blood glucose at a level above $100 \mathrm{mg} d \mathrm{~L}$. Patients should be closely monitored for minimum of 24 to 48 hours since hypoglycemia may recur after apparent clinical recovery. Clearance of GLUCOTROL from plasma would be prolonged in persons with liver disease. Because of the extensive protein binding of GLUCOTROL (glipizide), dialysis is unlikety to be of benet:

DOSAGE AND ADMINISTRATION: There is no fixed dosage regimen for the management of diabetes melitus with GLUCOTROL: in general, it should be given approximately 30 minutes before a meal to achieve the greatest reduction in postprandial hyperglycemia

Initial Dose: The recommended starting dose is $5 \mathrm{mg}$ betore breaktast. Geriatric patients or those with liver disease may be started on $25 \mathrm{mg}$. Dosage adjustments should ordinarily be in increments of $2.5-5 \mathrm{mg}$. as determined by blood glucose response. At least several days should elapse between titration steps.

Maximum Dose: The maximum recommended total daily dose is $40 \mathrm{mg}$

Maintenance: Some patients may be eftectively controlled on a once-a-day regimen, while others show better response with divided dosing. Total daily doses above $15 \mathrm{mg}$ should ordinarily be divided.

HOW SUPPLIED: GLUCOTROL is available as white, dye-free, scored diamond-shaped tablets imprinted as follows $5 \mathrm{mg}$ tablet-Pfizer 411 (NDC $5 \mathrm{mg}$ 0049-4110-66) Bottles of 100: $10 \mathrm{mg}$ tablet-Pfizer 412 (NDC $10 \mathrm{mg}$ 0049-4120-65) Botties of 100

CAUTION: Federal law prohibits dispensing without prescription.

More detailed protessional intormation available on request.
American Association of Retired Persons and the National Institute on Aging. The participants specifically investigated the role of aluminum and other heavy metals as possible contributors to Alzheimer's disease.

The fact that such a conference was called is interesting in itself. Many years ago, "food faddists" who claimed that aluminum had some negative effect on body functions were viewed with skepticism. But it is the unanswered questions and concerns raised by this hypothesis that initiated the conference. This blossoming of interest in nutrition has been a long time in coming.

\section{Physicians can recite by heart a litany of woes} about the current health care crisis in America. Mary Sacco, the 1982 Easter Seal poster child, and Francis Rockett, M.D., her neurosurgeon, told the tale of their own adversity before a hearing of the Senate Labor and Human Resources Committee.

It seems that Dr. Rockett has been forced to relocate his Massachusetts practice because he can no longer afford to pay the malpractice premiums on his "high-risk" specialty. And Mary and her family must decide whether to part with the physician who has treated Mary since infancy or to incur financial hardship to commute to wherever Dr. Rockett sets up practice.

Their story is not unusual. Unfortunately, many of you have probably heard of or experienced equally disturbing situations.

The incidence of lung cancer in white men has significantly decreased for the first time in five decades, according to the National Cancer Institute. The 4 percent reduction has finally emerged 20 years after the first large wave of white male smokers kicked the habit.

"This proves that people can successfully reduce their cancer risk by quitting smoking or not taking up smoking," said Vincent T. DeVita, Jr., M.D., director of the NCI.

Unfortunately, the outlook is not as optimistic for women or black men. Lung carcinoma will soon overtake breast carcinoma as the leading cause of cancer death in women. While new cases of lung cancer in black men appears to be leveling off, the incidence is still almost 60 percent higher than for white men. 



\section{WHEN EFFICACY IS YOUR FIRST CONCERN.}

There may be times when price and convenience are considerations in your choice of an NSAID. But when your primary concern is anti-arthritic efficacy, consider TOLECTIN DS tolmetin sodium.

TOLECTIN DS reduces arthritis pain and inflammation while improving functional capacity - as effectively as indomethacin. ${ }^{1}$

Yet, long-term, double-blind studies show that TOLECTIN DS, unlike indomethacin, rarely causes CNS side effects such as dizziness and light-headedness. The most frequent side effects were Gl related in both treatment groups.'

So, when your first concern is anti-arthritic efficacy, your first choice should be TOLECTIN DS-as effective as indomethacin, but often gentler.

1. Caldwell J, Brandon ML Franz KH et al: A double-blind comparison of the efficacy and side effect lia bility of tolmetin and indomethacin. Presented at a symposium, Tolmetin: A New Non-Steroidal AntiInflammatory Agent. Washington, DC, April 1975. Excerpta Medica, 1976, pp 57-70.

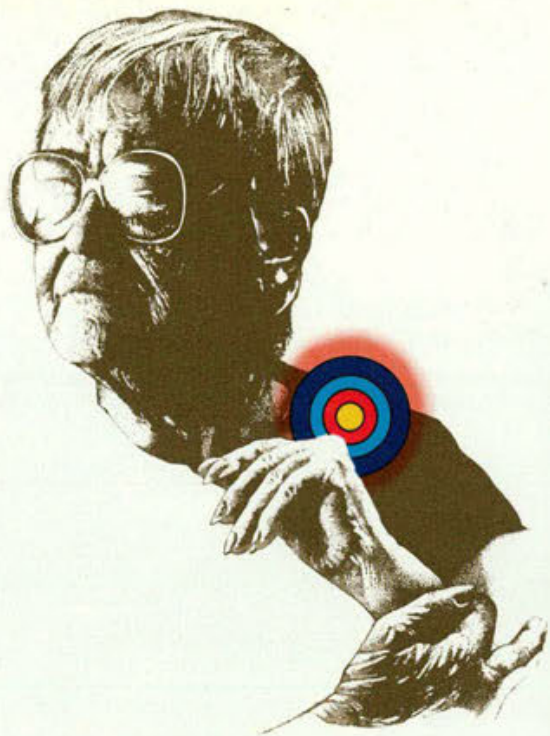

\section{For Arthritis}

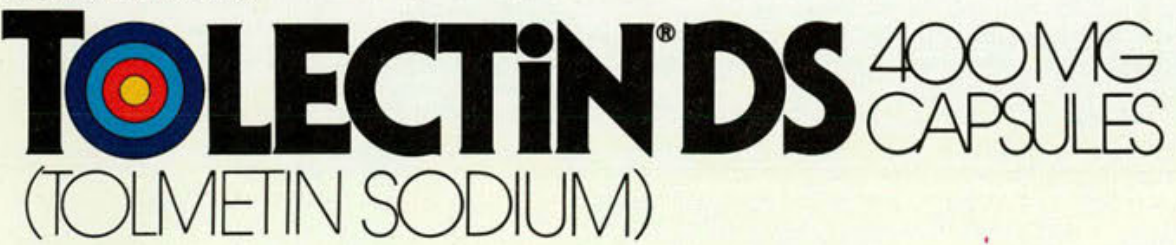

\section{ASEFFECTIVE AS INDOMETHACIN..OFTEN GENTLER}

\section{TOLECTIN (tolmetin sodium)}

The following is a brief summary only. Before prescribing, see complete prescribing information in TOLECTIN product labeling.

Contraindications: Anaphylactoid reactions have been reported with TOLECTIN as with other nonsteroidal anti-inflammatory drugs. Because of the possibility for cross-sensitivity. TOLECTIN should not be given to patients in whom aspirin and other nonsteroidal anti-inflammatory drugs (particularly zomepirac sodium) induce symptoms of asthma rhinitis, urticaria or other symptoms of allergic or anaphylactoid reactions. Patients experiencing anaphylactoid reactions on TOLECTIN should be treated with conventional therapy. such as epinephrine, antihistamine and/or steroids.

Warnings: Give under close supervision to patients with a history of upper gastrointestinal tract disease and only after consulting the

"Adverse Reactions" section. Peptic ulceration and gastrointestinal Adverse Reactions" section. Peptic ulceration and gastrointestinal given to patients with active peptic ulcer, closely supervise for signs of given to patients with active peptic ulcer, closely superisticer perforations or severe gastrointestinal bleeding

ulcer perforations or severe gastrointestinal bleeding. treatment with TOLECTIN should have ophthalmologic evaluations an follow-up

Cases of acute interstitial nephritis with hematuria, proteinuria, and occasionally nephrotic syndrome have been reported. Closely monitor patients with impaired renal function; they may require lower doses. In patients with prerenal conditions leading to a reduction of rena blood flow or blood volume administration of an NSAID may precipitate blood flow or blood volume, administration ol an NSAD may precipitate tailure, liver dysfunction, those taking diuretics, and the elderly.

TOLECTIN prolongs bleeding time. Patients who may be adversely
Tol affected by prolongation of bleeding time should be carefully observed when TOLECTIN is administered.

In patients receiving concomitant TOLECTIN-steroid therapy, any reduction in steroid dosage should be gradual to avoid the possible complications of sudden steroid withdrawal.

TOLECTIN should be used with caution in patients with compromised cardiac function, hypertension, or other conditions predisposing to fluid retention.

A patient with symptoms and/or signs suggesting liver dysfunction, or in whom an abnormal liver test has occurred, should be evaluated fo evidence of the development of more severe hepatic reactions while on therapy with TOLECTIN. Severe hepatic reactions, including jaundice and fatal hepatitis, have been reported with TOLECTIN as with other nonsteroidal anti-inflammatory drugs. Although such reactions are rare, if abnormal liver tests persist or worsen, it clinical signs and symptoms consistent with liver disease develop, or if systemic manifestations occur (e.g. eosinophilia, rash, etc.), discontinue TOLECTIN Carcinogenesis, Mutagenesis, Impairment of Festility - Tolmetin sodium did not possess any carcinogenic liability, mutagenic potential or impair nosss a carcinogenic liatily, mulagenic potent or impairment of tertility in standard in vitro tests and/or in tests in animals. Effects on parturition (including increased incidences of other prostaglandin inhibitors.

other prostagiandin inhibitors.
Pregnancy - TOLECTIN has not been studied in pregnant women. Pregnancy-TOLECTIN has not been studied in pregnant women
Orugs in this class have known effects on the fetal cardiovascular Drugs in this class have known effects on the fetal cardiovascular
system which may cause constriction of the ductus arteriosus in uter system which may cause constriction of the ductus arteriosus in utero pulmonary hypertension of the newborn. Therefore. TOLECTIN should be used during pregnancy only if the potential benefit justifies the potential risk to the fetus.

Nursing Mothers - Because TOLECTIN is secreted in human milk, nursing should not be undertaken while a patient is on this drug Pediatric Use - The safety and effectiveness of TOLECTIN for children under 2 years of age have not been established.

Drug Interactions - Increased prothrombin time and bleeding have Drug Interactions-Increased prothrombin time and bleeding have been reported in patients on concomitant TOLECTIN and warfarin

patients on anticoagulants.
Drug/Laboratory Test Interaction-Metabolites of tolmetin in urine have been found to give positive tests for proteinuria using tests which rely on acid precipitation as their endpoint. No interference is seen in the tests for proteinuria using dye-impregnated commercially available reagent strips.

Drug-Food Interaction - In a controlled single dose study, administration of TOLECTIN with milk had no effect on peak plasma tolmetin concentration, but decreased total tolmetin bioavailability by $16 \%$. When TOLECTIN was taken immediately after a meal, peak plasma tolmetin concentration and total bioavailability were reduced by $50 \%$ and $16 \%$, respectively.
Adverse Reactions: Incidence Greater Than $1 \%$-The follow adverse reactions which occurred more frequently than 1 in 100 wert reported in controlled clinical trials

Gastrointestinal: Nausea (11\%), dyspepsia, " gastrointestinal distress," abdominal pain," diarthea," flatulence," vomiting,"

constipation, gastritis, and peptic ulcer.

Body as a Whole: Headache," asthenia," chest pain Cardiovascular: Elevated blood pressure, "edema" Central Nervous System: Dizziness, "drowsiness, depression Metabolic/Nutritional: Weight gain," weight loss" Dermatologic: Skin irritation

Special Senses: Tinnitus, visual disturbance

Special Senses: Tinnitus, visual disturbance hematocrit not associated with gastrointestinal bleeding have occur Urogenital: Elevated BUN, urinary tract infection

- Reactions occurring in $3 \%$ to $9 \%$ of patients treated with TOLECT Reactions occurring in fewer than $3 \%$ of the patients are unmarked Incidence Less Than $1 \%$ (Causal Relationship Probable)

Gastrointestinal: Gastrointestinal bleeding with or without evidence peptic ulcer, glossitis, stomatitis, hepatitis, liver function abnormal Body as a Whole: Anaphylactoid reactions, tever, lymphadenopa serum sickness

Hematologic: Hemolytic anemia, thrombocytopenia, granulocyto penia, agranulocytosis

Cardiovascular: Congestive heart failure in patients with margin cardiac function

Dermatologic: Urticaria, purpura, erythema multiforme, toxic epidermal necrolysis

Urogenital: Hematuria, proteinuria, dysuria, renal failure Incidence Less Than 1\% (Causal Relationship Unknown)Body as a Whole: Epistaxis

Special Senses: Optic neuropathy, retinal and macular changes Full directions for use should be read before administering prescribing.

For information on symptoms and treatment of overdosage, see prescribing information. 